\title{
Free Vibration Frequency of Thick FGM Circular Cylindrical Shells with Simply Homogeneous Equation by Using TSDT
}

\author{
Chih-Chiang Hong* \\ Department of Mechanical Engineering, Hsiuping University of Science and Technology, Taichung, Taiwan \\ Received 04 June 2019; received in revised form 20 July 2019; accepted 17 September 2019 \\ DOI: https://doi.org/10.46604/aiti.2020.4380
}

\begin{abstract}
The objective of this study is to provide the frequency solutions of free vibration in thick FGM circular cylindrical shells by mainly considering both shear correction coefficient and nonlinear coefficient term. This paper investigates the effects of third-order shear deformation theory (TSDT) and the varied shear correction coefficient on the free vibration of thick functionally graded material (FGM), the circular cylindrical shells with simply homogeneous equation under thermal environment. The approach of derivations are given as follows, the varied value of shear correction coefficient is included in the simple homogeneous equation. The nonlinear term of displacement field of TSDT is also included to derive the simply homogeneous equation, some reasonable simplifications in the elements of homogeneous matrix under free vibration of thick FGM circular cylindrical shells are assumed, thus, the natural frequency can be found. Three parameters effect on the frequency of thick FGM circular cylindrical shells are computed and investigated, they are nonlinear coefficient $\boldsymbol{c}_{\boldsymbol{1}}$ term, environment temperature and power law index. There are some main conclusions obtained, generally the natural frequency results are in decreasing value with the mode shape numbers for the thicker circular cylindrical shells. The values of natural frequencies are also affected by the nonlinear coefficient term.
\end{abstract}

Keywords: third-order shear deformation theory, TSDT, FGM, shells, vibration, frequency

\section{Introduction}

There are some free vibration frequency investigations with shear deformation effect and experimental studies in the functionally graded material (FGM) circular cylindrical shells. In 2019, Shahbaztabar et al. [1] presented the free vibration of FGM circular cylindrical shells, including the Pasternak foundation and stationary fluid by using the first order shear deformation theory (FSDT). Some effects on the result of the natural frequencies are investigated e.g. fluid depth ratio, elastic foundation, volume fraction exponent, geometrical parameters and boundary conditions. In 2019, Zippo et al. [2] used the experimental method and the model of finite element method (FEM) to study the linear and dynamic behavior of vibrations in the polymeric circular cylindrical shell with FGM equivalent thermal temperature properties. In 2018, Baltacioğlu and Civalek [3] used the Love's shell theory and FSDT of the displacements to obtain the numerical results for the circular cylindrical FGM with carbon nanotube reinforced (CNTR) panels. For the thick FGM shells, it is necessary to consider the nonlinear terms of displacement theories to obtain more accurate results of analyses, e.g. third-order shear deformation theory (TSDT), higher-order shear deformation theory and triangular function shear deformation theory. In 2018, Torabi and Ansari [4] presented a formulation of higher-order isoparametric supplement to study the free vibration of FGM shells, considering the structural effects of circular cylindrical, conical, spherical and toroid shells. In 2017, Baltacioğlu and Civalek [5] used the extended FEM to obtain the frequency of the vibration of cracked FGM shells considering the structural effects of cylindrical

* Corresponding author. E-mail address: cchong@ mail.hust.edu.tw 
shell, conical shell and spherical on the vibrations. In 2017, Wang and Wu [6] presented the free vibration analysis of porous FGM cylindrical shells based on a sinusoidal shear deformation theory (SSDT). In 2014, Fazzolari and Carrera [7] presented the hierarchical trigonometric Ritz formulation (HTRF) and used in the free vibration analyses for the doubly curved FGM shells and sandwich shells with FGM core. In 2016, Fantuzzi et al. [8] presented free vibration analyses of cylindrical and spherical shells by using the FEM and the generalized differential quadrature (GDQ) methods. There are some new and improved TSDT used in the investigations of FGMs. In 2016, Bui et al. [9] presented the numerical results of deflection and frequency by using the TSDT and FEM for the static bending behaviors of FGM plates. A similar new TSDT in terms of five un-known variables was used in the eigenvalue equation to calculate the natural frequency. In 2017, Do et al. [10] presented the numerical results of deflection and stress by using the TSDT and FEM for the static buckling and bending behaviors of FGM plates. The same new TSDT in terms of five un-known variables was used in the bending equation and pre-buckling equation respectively to calculate the numerical solutions without considering the effect of shear correction factors. In 2018 , Vu et al. [11] presented the numerical results of deflection and frequency by using the TSDT and meshfree method for the static bending, free vibration and buckling behaviors of FGM plates. A similar refined TSDT in terms of four un-known variables was used in the equations to calculate the numerical solutions.

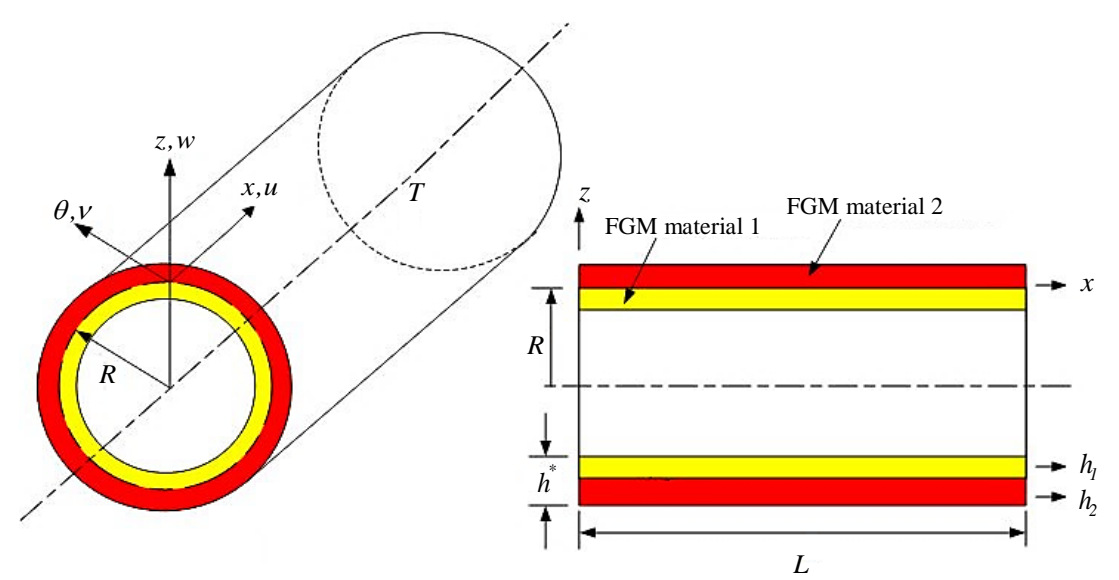

Fig. 1 Two-material thick FGM circular cylindrical shells

There are some importance and relevance of studied topics in the dynamics of cylindrical shells composed of FGMs. In 2012, Zhang et al. [12] presented the nonlinear dynamics of clamped-clamped FGM circular cylindrical shells under an external excitation and uniform temperature change. The similar equations were used and based on the FSDT and von-Karman nonlinear strains-displacement relation to obtain the numerical response of displacements. In 2016, Dai et al. [13] presented the reviews of coupled mechanics on the FGM cylindrical structures during years 2000-2015. Some of the existing mechanical theories and hypotheses were assumed and would be improved in the future for obtaining the more accuracy of the results. In 2008, Ansari and Darvizeh [14] presented a general analytical approach in arbitrary boundary conditions of FGM circular cylindrical shells. The FSDT of displacements was used to derive the homogeneous linear system and obtained the natural frequency under different boundary conditions. The author has some GDQ computational experiences in the composited FGM circular cylindrical shells. In 2017, Hong [15] used the approach of FSDT model and the varied shear correction factor to present the numerical GDQ results of thermal vibration and flutter of a supersonic air flowed over thick FGM circular cylindrical shells. In 2017, Hong [16] used the approach of Love's theory for thin multilayered shells to present the numerical GDQ results of displacement and stresses of thin FGM laminated magnetostrictive shells with the value effects of velocity feedback and control gain subjected to thermal vibration. It is interesting to investigate the natural frequency in the TSDT approach of thick FGM circular cylindrical shells under free vibration with simply homogeneous equation and four edges in simply supported boundary conditions. The value effects of three parametric: nonlinear coefficient $C_{l}$ term, environment temperature and power law index on the natural frequency of thick FGM circular cylindrical shells are investigated. The main contribution and novelty of paper is to provide and investigate the analytic solutions of natural frequencies in the free vibration 
of thick FGM circular cylindrical shells by considering the varied effects of shear correction coefficient and nonlinear terms of TSDT. The motivation of the paper is listed as follows. To obtain the natural frequency values of FGM shells considering the nonlinear coefficient terms of TSDT within the simply homogeneous matrix equation. Also by considering the calculated values of shear correction coefficient usually varied with thickness, power law index and environment temperature.

\section{Formulation}

For a two-material thick FGM circular cylindrical shells in thermal environment with thickness $h_{1}$ of FGM material 1 and thickness $h_{2}$ of FGM material 2. Fig. 1 shows a colorful figure that can illustrate the FGM material for the approach of the study. The material properties of power-law function of FGM circular cylindrical shells are considered with a Young's modulus $E_{f g m}$ of FGM in the standard variation form of power law index $R_{n}$, the others are assumed in the simple average form [17]. The properties $P_{i}$ of individual constituent material of FGMs are functions of environment temperature T in the following form [18],

$$
P_{i}=P_{0}\left(P_{-1} T^{-1}+1+P_{1} T+P_{2} T^{2}+P_{3} T^{3}\right)
$$

where $P_{0}, P_{-1}, P_{1}, P_{2}$ and $P_{3}$ are the temperature coefficients.

The time dependent of nonlinear displacements $u, v$ and $w$ of thick FGM circular cylindrical shells are assumed in the nonlinear coefficient $C_{1}$ term of TSDT equations [19] as follows,

$$
\begin{aligned}
& u=u_{0}(x, \theta, t)+z \phi_{x}(x, \theta, t)-c_{1} z^{3}\left(\phi_{x}+\frac{\partial w}{\partial x}\right) \\
& v=v_{0}(x, \theta, t)+z \phi_{\theta}(x, \theta, t)-c_{1} z^{3}\left(\phi_{\theta}+\frac{\partial w}{R \partial \theta}\right) \\
& w=w(x, \theta, t)
\end{aligned}
$$

where $u_{0}$ and $v_{0}$ are tangential displacements in the in-surface coordinates $x$ and $\theta$ axes direction, respectively, $w$ is transverse displacement in the out of surface coordinates $z$ axis direction of the middle-plane of circular cylindrical shells, $\phi_{x}$ and $\phi_{\theta}$ are the shear rotations, $R$ is the middle-surface radius of FGM shell, $t$ is time. Coefficient for $c_{1}=4 /\left(3 h^{* 2}\right)$ is given as in TSDT approach, in which $h^{*}$ is the total thickness of circular cylindrical shells. The linear time dependent of displacements also can be obtained by letting $c_{1}=0$ in the equations. The nonlinear coefficient $c_{1}$ term of displacement fields of TSDT [19] is used in the thick FGM circular cylindrical shells to investigate the nonlinear value effect on the natural frequency results. For the normal stresses $\left(\sigma_{x}\right.$ and $\left.\sigma_{\theta}\right)$ and the shear stresses $\left(\sigma_{x \theta}, \sigma_{\theta z}\right.$ and $\left.\sigma_{x z}\right)$ in the thick FGM circular cylindrical shells under temperature difference $\Delta T$ for the $k$ th layer are in the following equations [20-21],

$$
\begin{aligned}
& \left\{\begin{array}{c}
\sigma_{x} \\
\sigma_{\theta} \\
\sigma_{x \theta}
\end{array}\right\}_{(k)}=\left[\begin{array}{lll}
\bar{Q}_{11} & \bar{Q}_{12} & \bar{Q}_{16} \\
\bar{Q}_{12} & \bar{Q}_{22} & \bar{Q}_{26} \\
\bar{Q}_{16} & \bar{Q}_{26} & \bar{Q}_{66}
\end{array}\right]_{(k)}\left\{\begin{array}{c}
\varepsilon_{x}-\alpha_{x} \Delta T \\
\varepsilon_{\theta}-\alpha_{\theta} \Delta T \\
\varepsilon_{x \theta}-\alpha_{x \theta} \Delta T
\end{array}\right\}_{(k)}, \\
& \left\{\begin{array}{l}
\sigma_{\theta z} \\
\sigma_{x z}
\end{array}\right\}_{(k)}=\left[\begin{array}{ll}
\bar{Q}_{44} & \bar{Q}_{45} \\
\bar{Q}_{45} & \bar{Q}_{55}
\end{array}\right]_{(k)}\left\{\begin{array}{l}
\varepsilon_{\theta z} \\
\varepsilon_{x z}
\end{array}\right\}_{(k)},
\end{aligned}
$$

where $\alpha_{x}$ and $\alpha_{\theta}$ are the coefficients of thermal expansion, $\alpha_{x \theta}$ is the coefficient of thermal shear, $\bar{Q}_{i j}$ is the stiffness of FGM circular cylindrical shells. $\varepsilon_{x}, \varepsilon_{\theta}$ and $\varepsilon_{x \theta}$ are in-plane strains, not negligible $\varepsilon_{\theta z}$ and $\varepsilon_{x z}$ are shear strains. 
The dynamic equations of motion with TSDT for a thick FGM circular cylindrical shells are given as follows [22],

$$
\begin{aligned}
& \frac{\partial N_{x x}}{\partial x}+\frac{1}{R} \frac{\partial N_{x \theta}}{\partial \theta}=I_{0} \frac{\partial^{2} u_{0}}{\partial t^{2}}+J_{1} \frac{\partial^{2} \phi_{x}}{\partial t^{2}}-c_{1} I_{3} \frac{\partial^{2}}{\partial t^{2}}\left(\frac{\partial w}{\partial x}\right) \\
& \frac{\partial N_{x \theta}}{\partial x}+\frac{1}{R} \frac{\partial N_{\theta \theta}}{\partial \theta}=I_{0} \frac{\partial^{2} v_{0}}{\partial t^{2}}+J_{1} \frac{\partial^{2} \phi_{\theta}}{\partial t^{2}}-c_{1} I_{3} \frac{\partial^{2}}{\partial t^{2}}\left(\frac{\partial w}{R \partial \theta}\right) \\
& \frac{\partial \bar{Q}_{x}}{\partial x}+\frac{1}{R} \frac{\partial \bar{Q}_{\theta}}{\partial \theta}+c_{1}\left(\frac{\partial^{2} P_{x x}}{\partial x^{2}}+\frac{2}{R} \frac{\partial^{2} P_{x \theta}}{\partial x \partial \theta}+\frac{1}{R^{2}} \frac{\partial^{2} P_{\theta \theta}}{\partial \theta^{2}}\right)+q \\
& =I_{0} \frac{\partial^{2} w}{\partial t^{2}}-c_{1}^{2} I_{6} \frac{\partial^{2}}{\partial t^{2}}\left(\frac{\partial^{2} w}{\partial x^{2}}+\frac{1}{R^{2}} \frac{\partial^{2} w}{\partial \theta^{2}}\right)+c_{1}\left[I_{3} \frac{\partial^{2}}{\partial t^{2}}\left(\frac{\partial u_{0}}{\partial x}+\frac{1}{R} \frac{\partial v_{0}}{\partial \theta}\right)+J_{4} \frac{\partial^{2}}{\partial t^{2}}\left(\frac{\partial \phi_{x}}{\partial x}+\frac{1}{R} \frac{\partial \phi_{\theta}}{\partial \theta}\right)\right] \\
& \frac{\partial \bar{M}_{x x}}{\partial x}+\frac{1}{R} \frac{\partial \bar{M}_{x \theta}}{\partial \theta}-\bar{Q}_{x}=\frac{\partial^{2}}{\partial t^{2}}\left(J_{1} u_{0}+K_{2} \phi_{x}-c_{1} J_{4} \frac{\partial w}{\partial x}\right) \\
& \frac{\partial \bar{M}_{x \theta}}{\partial x}+\frac{1}{R} \frac{\partial \bar{M}_{\theta \theta}}{\partial \theta}-\bar{Q}_{\theta}=\frac{\partial^{2}}{\partial t^{2}}\left(J_{1} v_{0}+K_{2} \phi_{\theta}-c_{1} J_{4} \frac{1}{R} \frac{\partial w}{\partial \theta}\right)
\end{aligned}
$$

where

$$
\begin{aligned}
& \bar{M}_{\alpha \beta}=M_{\alpha \beta}-c_{1} P_{\alpha \beta} \\
& \bar{Q}_{\alpha}=Q_{\alpha}-c_{1} P_{\alpha},(\alpha, \beta=x, \theta) \\
& \left\{\begin{array}{l}
N_{x x} \\
N_{\theta \theta} \\
N_{x \theta}
\end{array}\right\}=\int_{-\frac{h^{*}}{2}}^{\frac{h^{*}}{2}}\left\{\begin{array}{l}
\sigma_{x} \\
\sigma_{\theta \theta} \\
\sigma_{x \theta}
\end{array}\right\} d z \\
& \left\{\begin{array}{l}
M_{x x} \\
M_{\theta \theta} \\
M_{x \theta}
\end{array}\right\}=\int_{-\frac{h^{*}}{2}}^{\frac{h^{*}}{2}}\left\{\begin{array}{l}
\sigma_{x} \\
\sigma_{\theta \theta} \\
\sigma_{x \theta}
\end{array}\right\} z d z \\
& \left\{\begin{array}{l}
P_{x x} \\
P_{\theta \theta} \\
P_{x \theta}
\end{array}\right\}=\int_{-\frac{h^{*}}{2}}^{\frac{h^{*}}{2}}\left\{\begin{array}{l}
\sigma_{x} \\
\sigma_{\theta \theta} \\
\sigma_{x \theta}
\end{array}\right\} z^{3} d z \\
& \left\{\begin{array}{l}
Q_{\theta} \\
Q_{x}
\end{array}\right\}=\int_{-\frac{h^{*}}{2}}^{\frac{h^{*}}{2}}\left\{\begin{array}{l}
\sigma_{\theta z} \\
\sigma_{x z}
\end{array}\right\} d z \\
& I_{i}=\sum_{k=1}^{N^{*}} \int_{k}^{k+1} \rho^{(k)} z^{i} d z,(i=0,1,2, \ldots, 6)
\end{aligned}
$$

where $N^{*}$ is the total number of layers, $\rho^{(k)}$ is the density of $k$ th ply. $J_{i}=I_{i}-c_{l} I_{i+2},(i=1,4), K_{2}=I_{2}-2 c_{1} I_{4}+c_{1}^{2} I_{6}$

There are some assumptions for the terms in the strains, e.g. the higher order terms $(\partial w / \partial x)^{2},[\partial w /(R \partial \theta)]^{2}$ and $(\partial w / \partial x)[\partial w /(R \partial \theta)]$ can't be neglected. The Von Karman type of strain-displacement relations with $\partial v_{0} / \partial z=-v_{0} / R$, $\partial u_{0} / \partial z=-u_{0} / R$ and $\partial w / \partial z=\partial \phi_{x} / \partial z=\partial \phi_{\theta} / \partial z=0$ are used as Eq. (6),

By substituting equations (3) and (6) into equation (4), the dynamic equilibrium differential equations with TSDT of thick FGM circular cylindrical shells in terms of partial derivatives of displacements and shear rotations subjected to the expressions terms $\left(f_{1}, \ldots, f_{5}\right)$ in partial derivatives of thermal loads $(\bar{N}, \bar{M}, \bar{P})$, mechanical loads $\left(p_{1}, p_{2}, q\right)$ and inertia terms can be derived and expressed in matrix forms. By assuming that mid-plane strain terms $(1 / 2)(\partial w / \partial x)^{2},(\partial w / \partial x)(1 / R)(\partial w / \partial \theta)$ and $(1 / 2)[(1 / R)(\partial w / \partial \theta)]^{2}$ are in constant values, the $f_{1}, \ldots, f_{5}$ can be expressed in the derivative terms as Eq. (7)-(8), 


$$
\begin{aligned}
\varepsilon_{x} & =\frac{\partial u}{\partial x}+\frac{1}{2}\left(\frac{\partial w}{\partial x}\right)^{2}=\frac{\partial u_{0}}{\partial x}+z \frac{\partial \phi_{x}}{\partial x}-c_{1} z^{3}\left(\frac{\partial \phi_{x}}{\partial x}+\frac{\partial^{2} w}{\partial x^{2}}\right)+\frac{1}{2}\left(\frac{\partial w}{\partial x}\right)^{2} \\
\varepsilon_{\theta} & =\frac{1}{R} \frac{\partial v}{\partial \theta}+\frac{1}{2}\left(\frac{1}{R} \frac{\partial w}{\partial \theta}\right)^{2}=\frac{1}{R} \frac{\partial v_{0}}{\partial \theta}+z \frac{1}{R} \frac{\partial \phi_{\theta}}{\partial \theta}-c_{1} z^{3}\left(\frac{1}{R} \frac{\partial \phi_{\theta}}{\partial \theta}+\frac{1}{R^{2}} \frac{\partial^{2} w}{\partial \theta^{2}}\right)+\frac{1}{2} \frac{1}{R^{2}}\left(\frac{\partial w}{\partial \theta}\right)^{2} \\
\varepsilon_{z z} & =0 \\
\varepsilon_{x \theta} & =\frac{1}{R} \frac{\partial u}{\partial \theta}+\frac{\partial v}{\partial x}+\left(\frac{\partial w}{\partial x}\right)\left(\frac{1}{R} \frac{\partial w}{\partial \theta}\right) \\
& =\frac{1}{R}\left[\frac{\partial u_{0}}{\partial \theta}+z \frac{\partial \phi_{x}}{\partial \theta}-c_{1} z^{3}\left(\frac{\partial \phi_{x}}{\partial \theta}+\frac{\partial^{2} w}{\partial x \partial \theta}\right)\right]+\frac{\partial v_{0}}{\partial x}+z \frac{\partial \phi_{\theta}}{\partial x}-c_{1} z^{3}\left(\frac{\partial \phi_{\theta}}{\partial x}+\frac{1}{R} \frac{\partial^{2} w}{\partial x \partial \theta}\right)+\left(\frac{\partial w}{\partial x}\right)\left(\frac{1}{R} \frac{\partial w}{\partial \theta}\right) \\
\varepsilon_{\theta z} & =\frac{\partial v}{\partial z}+\frac{1}{R} \frac{\partial w}{\partial \theta}=\frac{-v_{0}}{R}+\phi_{\theta}-3 c_{1} z^{2}\left(\phi_{\theta}+\frac{1}{R} \frac{\partial w}{\partial \theta}\right)+\frac{1}{R} \frac{\partial w}{\partial \theta} \\
\varepsilon_{x z} & =\frac{\partial u}{\partial z}+\frac{\partial w}{\partial x}=\frac{-u_{0}}{R}+\phi_{x}-3 c_{1} z^{2}\left(\phi_{x}+\frac{\partial w}{\partial x}\right)+\frac{\partial w}{\partial x} \\
f_{1} & =\frac{\partial \bar{N}_{x x}}{\partial x}+\frac{1}{R} \frac{\partial \bar{N}_{x \theta}}{\partial \theta}+p_{1} \\
f_{2} & =\frac{\partial \bar{N}_{x \theta}}{\partial x}+\frac{1}{R} \frac{\partial \bar{N}_{\theta \theta}}{\partial \theta}+p_{2} \\
f_{3} & =-q+c_{1}\left(\frac{\partial^{2} \bar{P}_{x x}}{\partial x^{2}}+\frac{2}{R} \frac{\partial^{2} \bar{P}_{x \theta}}{\partial x \partial \theta}+\frac{1}{R^{2}} \frac{\partial^{2} \bar{P}_{\theta \theta}}{\partial \theta^{2}}\right) \\
f_{4} & =\frac{\partial \bar{M}_{x x}}{\partial x}+\frac{1}{R} \frac{\partial \bar{M}_{x \theta}}{\partial \theta}-c_{1}\left(\frac{\partial \bar{P}_{x x}}{\partial x}+\frac{1}{R} \frac{\partial \bar{P}_{x \theta}}{\partial \theta}\right) \\
f_{5} & =\frac{\partial \bar{M}_{x \theta}}{\partial x}+\frac{1}{R} \frac{\partial \bar{M}_{\theta \theta}}{\partial \theta}-c_{1}\left(\frac{\partial \bar{P}_{x \theta}}{\partial x}+\frac{1}{R} \frac{\partial \bar{P}_{\theta \theta}}{\partial \theta}\right) \\
&
\end{aligned}
$$

and

$$
\begin{aligned}
& \left(\bar{N}_{x x}, \bar{M}_{x x}, \bar{P}_{x x}\right)=\int_{-\frac{h^{*}}{2}}^{\frac{h^{*}}{2}}\left(\bar{Q}_{11} \alpha_{x}+\bar{Q}_{12} \alpha_{\theta}+\bar{Q}_{16} \alpha_{x \theta}\right) \Delta T\left(1, z, z^{3}\right) d z \\
& \left(\bar{N}_{\theta \theta}, \bar{M}_{\theta \theta}, \bar{P}_{\theta \theta}\right)=\int_{-\frac{h^{*}}{2}}^{\frac{h^{*}}{2}}\left(\bar{Q}_{12} \alpha_{x}+\bar{Q}_{22} \alpha_{\theta}+\bar{Q}_{26} \alpha_{x \theta}\right) \Delta T\left(1, z, z^{3}\right) d z \\
& \left(\bar{N}_{x \theta}, \bar{M}_{x \theta}, \bar{P}_{x \theta}\right)=\int_{-\frac{h^{*}}{2}}^{\frac{h^{*}}{2}}\left(\bar{Q}_{16} \alpha_{x}+\bar{Q}_{26} \alpha_{\theta}+\bar{Q}_{66} \alpha_{x \theta}\right) \Delta T\left(1, z, z^{3}\right) d z \\
& \left(A_{i^{s} j^{s}}, B_{i^{s} j^{s}}, D_{i^{s} j^{s}}, E_{i^{s} j^{s}}, F_{i^{s} j^{s}}, H_{i^{s} j^{s}}\right)=\int_{-\frac{h^{*}}{2}}^{\frac{h^{*}}{2}} \bar{Q}_{i^{s} j^{s}}\left(1, z, z^{2}, z^{3}, z^{4}, z^{6}\right) d z,\left(i^{s}, j^{s}=1,2,6\right) \\
& \left(A_{i^{*} j^{*}}, B_{i^{*} j^{*}}, D_{i^{*} j^{*}}, E_{i^{*} j^{*}}, F_{i^{*} j^{*}}, H_{i^{*} j^{*}}\right)=\int_{-\frac{h^{*}}{2}}^{\frac{h^{*}}{2}} k_{\alpha} \bar{Q}_{i^{*} j^{*}}\left(1, z, z^{2}, z^{3}, z^{4}, z^{5}\right) d z,\left(i^{*}, j^{*}=4,5\right)
\end{aligned}
$$

where $p_{1}$ and $p_{2}$ are external in-plane distributed forces in $x$ and $\theta$ direction respectively. $q$ is external pressure load. $k_{\alpha}$ is the shear correction coefficient. $h^{*}$ is the total thickness of FGMs circular cylindrical shells. The computed and varied values of $k_{\alpha}$ are usually functions of total thickness of circular cylindrical shells, FGM power law index and environment temperature [23]. The $\bar{Q}_{i^{s} j^{s}}$ and $\bar{Q}_{i^{*} j^{*}}$ for thick FGM circular cylindrical shells with $z / R$ terms cannot be neglected are used in the following simple forms in 2014 by Hong [23], in 2010 by Sepiani et al. [24], 


$$
\begin{aligned}
& \bar{Q}_{11}=\bar{Q}_{22}=E_{f g m} /\left(1-v_{f g m}{ }^{2}\right) \\
& \bar{Q}_{12}=\bar{Q}_{21}=\left(v_{f g m} E_{f g m}\right) /\left[(1+z / R)\left(1-v_{f g m}{ }^{2}\right)\right] \\
& \bar{Q}_{44}=E_{f g m} /\left[2\left(1+v_{f g m}\right)\right] \\
& \bar{Q}_{55}=\bar{Q}_{66}=E_{f g m} /\left[2(1+z / R)\left(1+v_{f g m}\right)\right] \\
& \bar{Q}_{16}=\bar{Q}_{26}=\bar{Q}_{45}=0
\end{aligned}
$$

where $v_{f g m}=\left(v_{1}+v_{2}\right) / 2$ is the Poisson's ratios of the FGM circular cylindrical shells, $E_{f g m}=\left(E_{2}-E_{1}\right)\left[\left(z+h^{*}\right) / h^{*}\right]^{R_{n}}+E_{1}$ is the Young's modulus of the FGM circular cylindrical shells, $R_{n}$ is the power-law exponent parameter, $E_{1}$ and $E_{2}$ are the Young's modulus, $v_{1}$ and $v_{2}$ are the Poisson's ratios of the FGM constituent material 1 and 2, respectively. The simpler stiffness forms of $\bar{Q}_{i^{s} j^{s}}$ and $\bar{Q}_{i^{*} j^{*}}$ are used to calculate the stresses, $A_{i^{s} j^{s}}, B_{i^{s} j^{s}}, D_{i^{s} j^{s}}, E_{i^{s} j^{s}}, F_{i^{s} j^{s}}, H_{i^{s} j^{s}}$ and $A_{i^{*} j^{*}}, B_{i^{*} j^{*}}, D_{i^{*} j^{*}}, E_{i^{*} j^{*}}, F_{i^{*} j^{*}}, H_{i^{*} j^{*}}$. For example, by using change of variable in integration calculation, the $A_{11}, E_{11}, F_{11}, H_{11}$ and $H_{44}$ of thick FGM circular cylindrical shells are given as follows,

$$
\begin{aligned}
A_{11} & =\frac{h^{*}}{1-\left(\frac{v_{1}+v_{2}}{2}\right)^{2}}\left(\frac{R_{n} E_{1}+E_{2}}{R_{n}+1}\right) \\
E_{11}= & \frac{\left(h^{*}\right)^{4}\left(E_{2}-E_{1}\right)}{1-\left(\frac{v_{1}+v_{2}}{2}\right)^{2}}\left[\frac{1}{R_{n}+4}-\frac{3}{2\left(R_{n}+3\right)}+\frac{3}{4\left(R_{n}+2\right)}-\frac{1}{8\left(R_{n}+1\right)}\right] \\
F_{11}= & \frac{\left(h^{*}\right)^{5}}{1-\left(\frac{v_{1}+v_{2}}{2}\right)^{2}}\left\{\left(E_{2}-E_{1}\right)\left[\frac{1}{R_{n}+5}-\frac{2}{R_{n}+4}+\frac{1}{R_{n}+3}-\frac{1}{2\left(R_{n}+2\right)}+\frac{1}{16\left(R_{n}+1\right)}\right]+\frac{E_{1}}{80}\right\} \\
H_{11}= & \frac{\left(h^{*}\right)^{7}}{1-\left(\frac{v_{1}+v_{2}}{2}\right)^{2}}\left\{( E _ { 2 } - E _ { 1 } ) \left[\frac{1}{R_{n}+7}-\frac{3}{R_{n}+6}+\frac{13}{4\left(R_{n}+5\right)}-\frac{2}{R_{n}+4}+\frac{13}{16\left(R_{n}+3\right)}\right.\right. \\
& \left.\left.-\frac{3}{16\left(R_{n}+2\right)}+\frac{1}{64\left(R_{n}+1\right)}\right]+\frac{E_{1}}{448}\right\} \\
H_{44}= & \frac{k_{\alpha}\left(h^{*}\right)^{6}\left(E_{2}-E_{1}\right)}{2\left(1+\frac{v_{1}+v_{2}}{2}\right)}\left[\frac{1}{R_{n}+6}-\frac{5}{2\left(R_{n}+5\right)}+\frac{2}{R_{n}+4}-\frac{1}{R_{n}+3}+\frac{5}{64\left(R_{n}+2\right)}-\frac{1}{32\left(R_{n}+1\right)}\right]
\end{aligned}
$$

\section{Vibration Frequency}

The thick FGM circular cylindrical shells with layers in the stacking sequence $\left(0^{\circ} / 0^{\circ}\right)$ are used to study the free vibration frequency results with the effects of environment temperature and varied shear correction coefficient calculations, under four sides simply supported boundary condition, no thermal loads $(\Delta T=0)$, no in-plane distributed forces $\left(p_{1}=p_{2}=0\right)$ and no external pressure load $(q=0)$. The free vibration frequency $\omega_{m n}$ with mode shape numbers $m$ and $n$ for four sides simply supported boundary condition can be derived by simply assuming that $I_{1}=I_{3}=J_{1}=0, B_{i j}=E_{i j}=0, A_{16}=A_{26}=0$, $D_{16}=D_{26}=0$ and $A_{45}=D_{45}=F_{45}=0$ under the following time sinusoidal displacement and shear rotations forms with amplitudes $a_{m n}, b_{m n}, c_{m n}, d_{m n}$ and $e_{m n}$.

$$
\begin{aligned}
& u_{0}=a_{m n} \cos (m \pi x / L) \sin (n \pi \theta / R) \sin \left(\omega_{m n} t\right) \\
& v_{0}=b_{m n} \sin (m \pi x / L) \cos (n \pi \theta / R) \sin \left(\omega_{m n} t\right) \\
& w=c_{m n} \sin (m \pi x / L) \sin (n \pi \theta / R) \sin \left(\omega_{m n} t\right) \\
& \phi_{x}=d_{m n} \cos (m \pi x / L) \sin (n \pi \theta / R) \sin \left(\omega_{m n} t\right) \\
& \phi_{\theta}=e_{m n} \sin (m \pi x / L) \cos (n \pi \theta / R) \sin \left(\omega_{m n} t\right)
\end{aligned}
$$


where $m$ is the number of axial half-waves, $n$ is the number of circumferential waves. By substituting equations (11) into dynamic equilibrium differential equations under free vibration $\left(f_{1}=f_{2}=\ldots=f_{5}=0\right)$ with the assumed reasonable simplifications of $F H_{13}=F H_{14}=F H_{15}=F H_{23}=F H_{24}=F H_{25}=0$ and $I_{3}=J_{1}=I_{6}=J_{4}=0$ in the elements of homogeneous matrix, thus the simply homogeneous equation can be obtained as follows.

$$
\left[\begin{array}{ccccc}
F H_{11}-\lambda_{m n} & F H_{12} & 0 & 0 & 0 \\
F H_{12} & F H_{22}-\lambda_{m n} & 0 & 0 & 0 \\
0 & 0 & F H_{33}-\lambda_{m n} & F H_{34} & F H_{35} \\
0 & 0 & F H_{34} & F H_{44}-\frac{K_{2}}{I_{0}} \lambda_{m n} & F H_{45} \\
0 & 0 & F H_{35} & F H_{45} & F H_{55}-\frac{K_{2}}{I_{0}} \lambda_{m n}
\end{array}\right]\left\{\begin{array}{l}
a_{m n} \\
b_{m n} \\
c_{m n} \\
d_{m n} \\
e_{m n}
\end{array}\right\}=\left\{\begin{array}{l}
0 \\
0 \\
0 \\
0 \\
0
\end{array}\right\}
$$

where

$$
\begin{aligned}
\lambda_{m n}= & I_{0} \omega_{m n}{ }^{2} \\
F H_{11} & =A_{11}(m \pi / L)^{2}+A_{66}(n \pi / R)^{2} \\
F H_{12} & =\left(A_{12}+A_{66}\right)(m \pi / L)(n \pi / R) \\
F H_{22} & =A_{66}(m \pi / L)^{2}+A_{22}(n \pi / R)^{2} \\
F H_{33} & =A_{55}(m \pi / L)^{2}+A_{44}(n \pi / R)^{2}+c_{1}^{2} H_{11}(m \pi / L)^{4}+\left(2 c_{1}^{2} H_{12}+4 c_{1}^{2} H_{66}\right)(m \pi / L)^{2}(n \pi / R)^{2} \\
& +c_{1}{ }^{2} H_{22}(n \pi / R)^{4}-3 c_{1}\left(2 D_{55}-3 c_{1} F_{55}\right)(m \pi / L)^{2}-3 c_{1}\left(2 D_{44}-3 c_{1} F_{44}\right)(n \pi / R)^{2}
\end{aligned}
$$

and

$$
\begin{aligned}
F H_{34} & =A_{55}(m \pi / L)-\left(c_{1} F_{11}-c_{1}^{2} H_{11}\right)(m \pi / L)^{3}-\left(2 c_{1} F_{66}-3 c_{1}^{2} H_{66}+c_{1} F_{12}-c_{1}^{2} H_{12}\right)(m \pi / L)(n \pi / R)^{2} \\
& -\left(6 c_{1} D_{55}-9 c_{1}^{2} F_{55}\right)(m \pi / L) \\
F H_{35} & =A_{44}(n \pi / R)-\left(c_{1} F_{22}-c_{1}^{2} H_{22}\right)(n \pi / R)^{3}-\left(2 c_{1} F_{66}-3 c_{1}^{2} H_{66}+c_{1} F_{12}-c_{1}^{2} H_{12}\right)(m \pi / L)^{2}(n \pi / R) \\
& -\left(6 c_{1} D_{44}-9 c_{1}^{2} F_{44}\right)(n \pi / R) \\
F H_{44} & =\left(D_{11}-2 c_{1} F_{11}+c_{1}^{2} H_{11}\right)(m \pi / L)^{3}+\left(D_{66}-2 c_{1}^{2} F_{66}+c_{1}^{2} H_{66}\right)(n \pi / R)^{2}+A_{55}-6 c_{1} D_{55}+9 c_{1}^{2} F_{55} \\
F H_{45} & =\left(D_{12}+D_{66}-2 c_{1} F_{12}+c_{1}^{2} H_{12}-2 c_{1} F_{66}+c_{1}^{2} H_{66}\right)(m \pi / L)(n \pi / R) \\
F H_{55} & =\left(D_{66}-2 c_{1} F_{66}+c_{1}^{2} H_{66}\right)(m \pi / L)^{2}+\left(D_{22}-2 c_{1} F_{22}+c_{1}^{2} H_{22}\right)(n \pi / R)^{2}+A_{44}-6 c_{1} D_{44}+9 c_{1}^{2} F_{44}
\end{aligned}
$$

The determinant of the coefficient matrix in equation (12) vanishes for obtaining non-trivial solution of amplitudes can be represented in the simply five degree polynomial equation as follows, thus the $\omega_{m n}$ can be found.

$$
A(1) \lambda_{m n}{ }^{5}+A(2) \lambda_{m n}{ }^{4}+A(3) \lambda_{m n}{ }^{3}+A(4) \lambda_{m n}{ }^{2}+A(5) \lambda_{m n}+A(6)=0
$$

where

$$
\begin{aligned}
& A(1)=-s d \\
& A(2)=\left(F H_{11}+F H_{12}\right) s d+s c \\
& A(3)=-\left[\left(F H_{11} F H_{22}-F H_{12} F H_{12}\right) s d+\left(F H_{11}+F H_{12}\right) s c+s b\right] \\
& A(4)=\left(F H_{11} F H_{22}-F H_{12} F H_{12}\right) s c+\left(F H_{11}+F H_{22}\right) s b+s a \\
& A(5)=-\left[\left(F H_{11} F H_{22}-F H_{12} F H_{12}\right) s b+\left(F H_{11}+F H_{22}\right) s a\right] \\
& A(6)=\left(F H_{11} F H_{22}-F H_{12} F H_{12}\right) s a
\end{aligned}
$$


in which

$$
\begin{aligned}
s d & =\left(K_{2} / I_{0}\right)^{2} \\
s c & =F H_{33} s d+F H_{44} K_{2} / I_{0} \\
s b & =\left(F H_{33} F H_{55}+F H_{44} F H_{55}+F H_{33} F H_{44}-F H_{35} F H_{35}-F H_{34} F H_{34}\right) K_{2} / I_{0}-F H_{45} F H_{45} \\
s a & =F H_{33} F H_{44} F H_{55}+F H_{44} F H_{34} F H_{35}+F H_{35} F H_{34} F H_{45}-F H_{35} F H_{35} F H_{44}-F H_{34} F H_{34} F H_{55} \\
& -F H_{45} F H_{45} F H_{33}
\end{aligned}
$$

\begin{tabular}{|c|c|c|c|c|c|c|c|}
\hline \multirow{3}{*}{$L / h^{*}$} & \multirow{3}{*}{$R_{n}$} & \multirow{3}{*}{$\begin{array}{c}c_{1} \\
\left(1 / \mathrm{mm}^{2}\right)\end{array}$} & \multicolumn{5}{|c|}{$f^{*}$} \\
\hline & & & \multicolumn{5}{|c|}{ Present solution, $h^{*}=1.2 \mathrm{~mm}$, varied $k_{\alpha}$} \\
\hline & & & $T=1 K$ & $T=100 K$ & $T=300 K$ & $T=600 \mathrm{~K}$ & $T=1000 \mathrm{~K}$ \\
\hline \multirow{5}{*}{5} & 0.5 & 0.925925 & $\begin{array}{l}3.178485 \\
8.567362\end{array}$ & $\begin{array}{l}3.434401 \\
9391482\end{array}$ & $\begin{array}{c}3.873990 \\
10984527\end{array}$ & 4.111627 & $\begin{array}{r}3.662770 \\
135387665\end{array}$ \\
\hline & 1 & $\begin{array}{c}0.925925 \\
0\end{array}$ & $\begin{array}{l}3.318237 \\
7.958229\end{array}$ & $\begin{array}{l}3.570262 \\
8.647485\end{array}$ & $\begin{array}{l}4.005848 \\
9.985663\end{array}$ & $\begin{array}{l}4.253132 \\
11.39358\end{array}$ & $\begin{array}{c}3.862303 \\
12.597763\end{array}$ \\
\hline & 2 & 0.925925 & $\begin{array}{l}3.477849 \\
7132526\end{array}$ & 3.723252 & 4.151720 & 4.409905 & 4.098108 \\
\hline & \multirow{2}{*}{10} & 0.925925 & $\begin{array}{l}1.152520 \\
3.756482\end{array}$ & $\begin{array}{l}1.090011 \\
3.984509\end{array}$ & $\begin{array}{l}8 . / 63615 \\
4.394140\end{array}$ & $\frac{9.831241}{4.670961}$ & $\begin{array}{c}10.888006 \\
4.532945\end{array}$ \\
\hline & & 0 & 6.452526 & 6.885567 & 7.695008 & 8.305713 & 8.563466 \\
\hline \multirow{8}{*}{8} & \multirow{2}{*}{0.5} & 0.925925 & 2.238668 & 2.419486 & 2.730914 & 2.897884 & 2.576800 \\
\hline & & 0 & 6.204801 & 6.788758 & 7.907682 & 9.025515 & 9.594029 \\
\hline & \multirow[t]{2}{*}{1} & 0.925925 & 2.336488 & 2.514541 & 2.823131 & 2.996852 & 2.716518 \\
\hline & & 0 & 5.856612 & 6.350593 & 7.296222 & 8.251528 & 8.981841 \\
\hline & \multirow{2}{*}{2} & 0.925925 & 2.448283 & 2.621661 & 2.925246 & 3.106589 & 2.881696 \\
\hline & & 0 & 5.856612 & 6.350593 & 6.587167 & 7.313977 & 7.905719 \\
\hline & \multirow{2}{*}{10} & 0.925925 & 2.643672 & 2.804862 & 3.095301 & 3.289678 & 3.186499 \\
\hline & & 0 & 5.107512 & 5.428395 & 6.021389 & 6.482052 & 6.638852 \\
\hline \multirow{8}{*}{10} & \multirow{2}{*}{0.5} & 0.925925 & 1.926014 & 2.081703 & 2.349735 & 2.830540 & 2.217255 \\
\hline & & 0 & 5.478031 & 5.986835 & 6.954710 & 7.897100 & 8.325227 \\
\hline & \multirow{2}{*}{1} & 0.925925 & 2.010152 & 2.163467 & 2.429062 & 2.578504 & 2.337450 \\
\hline & & 0 & 5.224647 & 5.659943 & 6.485115 & 7.288469 & 7.833433 \\
\hline & \multirow[b]{2}{*}{2} & 0.925925 & 2.106326 & 2.255628 & 2.663339 & 2.672919 & 2.479556 \\
\hline & & 0 & 4.906995 & 5.272914 & 5.956491 & 6.573925 & 6.987514 \\
\hline & \multirow{2}{*}{10} & 0.925925 & 2.274471 & 2.413313 & 2.663339 & 2.830540 & 2.741827 \\
\hline & & 0 & 4.730258 & 5.024359 & 5.565807 & 5.980343 & 6.078461 \\
\hline
\end{tabular}

\section{Results and Discussion}

Table $1 f^{*}$ for SUS304/Si ${ }_{3} \mathrm{~N}_{4}$

The composited thick FGM SUS304/Si ${ }_{3} \mathrm{~N}_{4}$ material is used to implement the numerical computation of vibration under environment temperature $T$ (free stress assumed). The FGM material 1 at inner position of circular cylindrical shells is SUS304 (stainless steel), the FGM material 2 at outer position of circular cylindrical shells is $\mathrm{Si}_{3} \mathrm{~N}_{4}$ (silicon nitride) used for the free vibration frequency computations with simply homogeneous equation. For the preliminary FGM circular cylindrical shells study, it did not considered the effect of nonlinear coefficient term on the calculation of varied shear correction coefficient. The varied values of $k_{\alpha}$ are usually functions of $h^{*}, R_{n}$ and $T$ in the thick FGM circular cylindrical shells $\left(B_{i j} \neq 0\right)$. For $L / R=1$, $h_{1}=h_{2}, h^{*}=1.2 \mathrm{~mm}$, calculated values of $k_{\alpha}$ are increasing with $R_{n}$ (from 0.1 to 10). Thus values of $k_{\alpha}$ are used for frequency calculations of the free vibration (no thermal loads under no temperature difference $(\Delta T=0)$ including the effects of nonlinear coefficient $c_{1}$ term. Firstly, for the frequency parameter $f^{*}=4 \pi \omega_{11} R \sqrt{I_{2} / A_{11}}$ values under the effects of $c_{1}=$ $0.925925 / \mathrm{mm}^{2}$ and $c_{1}=0 / \mathrm{mm}^{2}$ for $L / h^{*}=5,8$ and 10 are shown in Table 1 , where $\omega_{11}$ is the fundamental first natural frequency $(m=n=1)$. For SUS304/Si ${ }_{3} \mathrm{~N}_{4}$ thick circular cylindrical shells under free vibration with $h^{*}=1.2 \mathrm{~mm}$, the $f^{*}$ values under $T=1 \mathrm{~K}, 100 \mathrm{~K}, 300 \mathrm{~K}, 600 \mathrm{~K}$ and $1000 \mathrm{~K}$ with varied $k_{\alpha}$ and $c_{1}$ effects are in the values not greater than 13.538765 . 
The frequency parameter $\Omega=\left(\omega_{11} L^{2} / h^{*}\right) \sqrt{\rho_{1} / E_{1}}$ values under the effects of $c_{1}=0.925925 / \mathrm{mm}^{2}$ and $c_{1}=0 / \mathrm{mm}^{2}$ for $L / h^{*}=$ 5, 8 and 10 are shown in Table 2, $\rho_{1}$ is the density of FGM material 1, for $\mathrm{SUS} 304 / \mathrm{Si}_{3} \mathrm{~N}_{4}$ thick circular cylindrical shells under free vibration with $h^{*}=1.2 \mathrm{~mm}$, the $\Omega$ values under $T=1 \mathrm{~K}, 100 \mathrm{~K}, 300 \mathrm{~K}, 600 \mathrm{~K}$ and $1000 \mathrm{~K}$ with varied $k_{\alpha}$ and $c_{1}$ effects are in the values not greater than 32.380783 .

It is easy to judge the machining abnormality by the external sensor signal in this experiment. At this time, the internal condition data could be combined to determine the type of abnormality as chatter. The accuracy of the monitoring is improved. There are a variety of visualization methods in this monitoring system, which could help to quickly locate abnormal positions. In addition, it helped to grasp the information of CNC machine tool conditions at all times, which reduced the difficulty of subsequent fault analysis and diagnosis.

Table $2 \Omega$ for SUS304/ $\mathrm{Si}_{3} \mathrm{~N}_{4}$

\begin{tabular}{|c|c|c|c|c|c|c|c|}
\hline \multirow{3}{*}{$L / h^{*}$} & \multirow{3}{*}{$R_{n}$} & \multirow{3}{*}{$\begin{array}{c}c_{1} \\
\left(1 / \mathrm{mm}^{2}\right)\end{array}$} & \multicolumn{5}{|c|}{$\Omega$} \\
\hline & & & \multicolumn{5}{|c|}{ Present solution, $h^{*}=1.2 \mathrm{~mm}$, varied $k_{\alpha}$} \\
\hline & & & $T=1 K$ & $T=100 K$ & $T=300 K$ & $T=600 K$ & $T=1000 K$ \\
\hline \multirow{4}{*}{5} & 0.5 & $\begin{array}{c}0.925925 \\
0\end{array}$ & $\begin{array}{c}5.781036 \\
15.582337\end{array}$ & $\begin{array}{c}6.101545 \\
16.684873 \\
\end{array}$ & $\begin{array}{c}6.693336 \\
18.978658 \\
\end{array}$ & $\begin{array}{c}7.132169 \\
21.886919\end{array}$ & $\begin{array}{c}7.123131 \\
26.329359\end{array}$ \\
\hline & 1 & $\begin{array}{l}0.925925 \\
0\end{array}$ & $\begin{array}{c}5.782522 \\
13.868399\end{array}$ & $\begin{array}{c}6.103089 \\
14.782213\end{array}$ & $\begin{array}{c}6.694982 \\
16.689058\end{array}$ & $\begin{array}{c}7.133930 \\
19.110862\end{array}$ & $\begin{array}{c}7.124928 \\
23.239543\end{array}$ \\
\hline & 2 & $\begin{array}{c}0.925925 \\
0\end{array}$ & $\begin{array}{c}5.783707 \\
11.861481\end{array}$ & $\begin{array}{c}6.104272 \\
12.608768\end{array}$ & $\begin{array}{c}6.696166 \\
14.134532\end{array}$ & $\begin{array}{c}7.135265 \\
15.907033\end{array}$ & $\begin{array}{c}7.126575 \\
18.934150\end{array}$ \\
\hline & 10 & $\begin{array}{c}0.925925 \\
0 \\
\end{array}$ & $\begin{array}{l}5.784393 \\
9.935876 \\
\end{array}$ & $\begin{array}{c}6.104699 \\
10.549432 \\
\end{array}$ & $\begin{array}{c}6.696107 \\
11.726208 \\
\end{array}$ & $\begin{array}{c}7.135397 \\
12.687871 \\
\end{array}$ & $\begin{array}{c}7.128233 \\
13.466383 \\
\end{array}$ \\
\hline \multirow{3}{*}{$L / h^{*}$} & \multirow{3}{*}{$R_{n}$} & \multirow{3}{*}{$\begin{array}{c}c_{1} \\
\left(1 / \mathrm{mm}^{2}\right)\end{array}$} & \multicolumn{5}{|c|}{$\Omega$} \\
\hline & & & \multicolumn{5}{|c|}{ Present solution, $h^{*}=1.2 \mathrm{~mm}$, varied $k_{\alpha}$} \\
\hline & & & $T=1 K$ & $T=100 K$ & $T=300 K$ & $T=600 K$ & $T=1000 K$ \\
\hline \multirow{4}{*}{8} & 0.5 & $\begin{array}{c}0.925925 \\
0 \\
\end{array}$ & $\begin{array}{c}6.514712 \\
18.056488 \\
\end{array}$ & $\begin{array}{c}6.877522 \\
19.297414 \\
\end{array}$ & $\begin{array}{c}7.549395 \\
21.860160 \\
\end{array}$ & $\begin{array}{c}8.042832 \\
25.049551 \\
\end{array}$ & $\begin{array}{c}8.017926 \\
29.852609 \\
\end{array}$ \\
\hline & 1 & $\begin{array}{l}0.925925 \\
0\end{array}$ & $\begin{array}{c}6.514685 \\
16.329633\end{array}$ & $\begin{array}{c}6.877464 \\
17.369365\end{array}$ & $\begin{array}{c}7.549288 \\
19.510704\end{array}$ & $\begin{array}{c}8.042762 \\
22.144931\end{array}$ & $\begin{array}{c}8.018013 \\
26.510597\end{array}$ \\
\hline & 2 & $\begin{array}{c}0.925925 \\
0 \\
\end{array}$ & $\begin{array}{c}6.514442 \\
16.329633 \\
\end{array}$ & $\begin{array}{c}6.877144 \\
17.369365 \\
\end{array}$ & $\begin{array}{c}7.548845 \\
16.998745 \\
\end{array}$ & $\begin{array}{c}8.042382 \\
18.934526 \\
\end{array}$ & $\begin{array}{c}8.017992 \\
21.996770 \\
\end{array}$ \\
\hline & 10 & $\begin{array}{c}0.925925 \\
0 \\
\end{array}$ & $\begin{array}{c}6.513346 \\
12.583626 \\
\end{array}$ & $\begin{array}{c}6.875763 \\
13.307022 \\
\end{array}$ & $\begin{array}{c}7.546949 \\
14.681323 \\
\end{array}$ & $\begin{array}{c}8.040542 \\
15.843253 \\
\end{array}$ & $\begin{array}{c}8.017431 \\
16.703767 \\
\end{array}$ \\
\hline \multirow{4}{*}{10} & 0.5 & $\begin{array}{c}0.925925 \\
0 \\
\end{array}$ & $\begin{array}{c}7.006078 \\
19.926910 \\
\end{array}$ & $\begin{array}{c}7.396695 \\
21.272380 \\
\end{array}$ & $\begin{array}{c}8.119573 \\
24.032178 \\
\end{array}$ & $\begin{array}{c}8.647910 \\
27.397165 \\
\end{array}$ & $\begin{array}{c}8.623964 \\
32.380783\end{array}$ \\
\hline & 1 & $\begin{array}{c}0.925925 \\
0\end{array}$ & $\begin{array}{c}7.005980 \\
18.209449\end{array}$ & $\begin{array}{c}7.396562 \\
19.350477\end{array}$ & $\begin{array}{c}8.119391 \\
21.677169\end{array}$ & $\begin{array}{c}8.650032 \\
24.450416\end{array}$ & $\begin{array}{c}8.623955 \\
28.901228\end{array}$ \\
\hline & 2 & $\begin{array}{c}0.925925 \\
0 \\
\end{array}$ & $\begin{array}{c}7.005695 \\
16.320789 \\
\end{array}$ & $\begin{array}{c}7.396207 \\
17.289890 \\
\end{array}$ & $\begin{array}{c}8.117177 \\
19.214038 \\
\end{array}$ & $\begin{array}{c}8.649613 \\
21.273336 \\
\end{array}$ & $\begin{array}{c}8.623854 \\
24.302459 \\
\end{array}$ \\
\hline & 10 & $\begin{array}{c}0.925925 \\
0\end{array}$ & $\begin{array}{c}7.004657 \\
14.567711\end{array}$ & $\begin{array}{c}7.394913 \\
15.395722\end{array}$ & $\begin{array}{c}8.117177 \\
16.963157\end{array}$ & $\begin{array}{c}8.647910 \\
18.271238\end{array}$ & $\begin{array}{c}8.623262 \\
19.117235\end{array}$ \\
\hline
\end{tabular}

It is interesting to compare the present vibration values of frequency with some authors' work as shown in the Tables (3)-(4). The values of $f^{*}$ vs. $h^{*}$ for SUS304/Si $\mathrm{N}_{4}$ under $L / h^{*}=10$ and $T=300 K$ with varied $k_{\alpha}$ and $c_{1}$ effects are shown in Table 3. The compared value $f^{*}=8.426538$ at $h^{*}=2 \mathrm{~mm}, R_{n}=0.5$ is greater than $f^{*}=8.0$ at $n=13$ with silicon nitride-nickel under classical shell theory (CST), no external pressure $\left(K_{e}=0\right)$ by Sepiani et al. in 2010 [24]. The values of $\Omega$ vs. $h^{*}$ for SUS304/Si $\mathrm{N}_{4}$ under $L / h^{*}=10$ and $T=700 \mathrm{~K}$ with varied $k_{\alpha}$ and $c_{1}$ effects are shown in Table 4 . The compared value $\Omega=$ 2.459972 at $c_{1}=0.925925 / \mathrm{mm}^{2}, h^{*}=1.2 \mathrm{~mm}, R_{n}=0.5$ is greater than $\Omega=1.71137$ with the material variation type A, three layers thickness ratio $1-8-1$, the L directional radius of curvature is $\infty, L / h^{*}=10, R_{n}=0.5$ for the FGM sandwich shell presented by Chen et al. in 2017 [25]. 
Table 3 Comparison of frequency $f^{*}$ for $\mathrm{SUS} 304 / \mathrm{Si}_{3} \mathrm{~N}_{4}$ and silicon nitride-nickel

\begin{tabular}{|c|c|c|c|c|c|}
\hline \multirow{3}{*}{$c_{1}\left(1 / \mathrm{mm}^{2}\right)$} & \multirow{3}{*}{$h^{*}(\mathrm{~mm})$} & \multicolumn{4}{|c|}{$f^{*}$} \\
\hline & & \multicolumn{3}{|c|}{$\begin{array}{c}\text { Present method, } L / h^{*}=10, T=300 K \\
\text { varied } k_{\alpha} \text {, for } S U S 304 / S_{i} N_{4}\end{array}$} & $\begin{array}{c}\text { Sepiani et al. 2010, } \\
\text { for silicon nitride-nickel, } \\
n=13\end{array}$ \\
\hline & & $R_{n}=0.5$ & $R_{n}=1$ & $R_{n}=2$ & - \\
\hline 0.925925 & 1.2 & 2.349735 & 2.429062 & 2.516924 & - \\
\hline 0.333333 & 2 & 8.426538 & 8.711254 & 9.026930 & 8.0 \\
\hline 0.000033 & 200 & 842669.2 & 871142.9 & 902712.1 & - \\
\hline 0.000014 & 300 & 253980.0 & 262560.1 & 272073.1 & - \\
\hline 0.000003 & 600 & 18903.19 & 19542.01 & 20250.14 & - \\
\hline 0.000001 & 900 & 43930.59 & 45414.97 & 47060.53 & - \\
\hline
\end{tabular}

Table 4 Comparison of frequency $\Omega$ for SUS304/Si ${ }_{3} \mathrm{~N}_{4}$

\begin{tabular}{|c|c|c|c|c|c|}
\hline \multirow{2}{*}{$c_{1}(1 / \mathrm{mm} 2)$} & \multirow{3}{*}{$h^{*}(\mathrm{~mm})$} & \multicolumn{4}{|c|}{$\Omega$} \\
\cline { 3 - 6 } & & \multicolumn{2}{|c|}{$\begin{array}{c}\text { Present method, } L / h^{*}=10, \\
\text { nyyyy}\end{array}$} & & \multicolumn{2}{|c|}{$T=700 K$, varied $k_{\alpha}$} & $\begin{array}{c}\text { Chen et al. 2017, type A, } \\
1-8-1, R_{n}=0.5\end{array}$ \\
\cline { 3 - 6 } & & $R_{n}=0.5$ & $R_{n}=1$ & $R_{n}=2$ & - \\
\hline 0.925925 & 1.2 & 2.459972 & 2.550420 & 2.651571 & 1.71137 \\
\hline 0.333333 & 2 & 8.821661 & 9.146189 & 9.509387 & - \\
\hline 0.000033 & 200 & 882174.8 & 914629.0 & 950949.6 & - \\
\hline 0.000014 & 300 & 267903.0 & 277757.0 & 288785.0 & - \\
\hline 0.000003 & 600 & 19897.76 & 20629.82 & 21448.99 & - \\
\hline 0.000001 & 900 & 46307.33 & 48010.84 & 49917.17 & \\
\hline
\end{tabular}

Table 5 Fundamental natural frequency $\omega_{11}$ for $h^{*}=1.2 \mathrm{~mm}$

\begin{tabular}{|c|c|c|c|c|c|c|c|}
\hline \multirow{2}{*}{$L / h^{*}$} & \multirow{2}{*}{$R_{n}$} & \multirow{2}{*}{$c_{1}\left(1 / \mathrm{mm}^{2}\right)$} & \multicolumn{5}{|c|}{$\omega_{11}$} \\
\hline & & & $T=1 K$ & $T=100 K$ & $T=300 K$ & $T=600 K$ & $T=1000 K$ \\
\hline \multirow{8}{*}{5} & \multirow{2}{*}{0.5} & 0.925925 & 0.001620 & 0.001730 & 0.001906 & 0.001947 & 0.001614 \\
\hline & & 0 & 0.004366 & 0.004731 & 0.005406 & 0.005975 & 0.005968 \\
\hline & \multirow{2}{*}{1} & 0.925925 & 0.001620 & 0.001730 & 0.001907 & 0.001947 & 0.001615 \\
\hline & & 0 & 0.003886 & 0.004191 & 0.004753 & 0.005217 & 0.005267 \\
\hline & \multirow{2}{*}{2} & 0.925925 & 0.001620 & 0.001730 & 0.001907 & 0.001948 & 0.001615 \\
\hline & & 0 & 0.003324 & 0.003575 & 0.004026 & 0.004343 & 0.004291 \\
\hline & \multirow{2}{*}{10} & 0.925925 & 0.001620 & 0.001731 & 0.001907 & 0.001948 & 0.001615 \\
\hline & & 0 & 0.002784 & 0.002991 & 0.003340 & 0.003464 & 0.003052 \\
\hline \multirow{8}{*}{8} & \multirow{2}{*}{0.5} & 0.925925 & 0.000713 & 0.000761 & 0.000840 & 0.000857 & 0.000709 \\
\hline & & 0 & 0.001976 & 0.002137 & 0.002432 & 0.002671 & 0.002643 \\
\hline & \multirow{2}{*}{1} & 0.925925 & 0.000713 & 0.000761 & 0.000840 & 0.000857 & 0.000709 \\
\hline & & 0 & 0.001787 & 0.001924 & 0.002170 & 0.002361 & 0.002347 \\
\hline & \multirow[b]{2}{*}{2} & 0.925925 & 0.000713 & 0.000761 & 0.000839 & 0.000857 & 0.000709 \\
\hline & & 0 & 0.001787 & 0.001924 & 0.001891 & 0.002019 & 0.001947 \\
\hline & \multirow{2}{*}{10} & 0.925925 & 0.000712 & 0.000761 & 0.000839 & 0.000857 & 0.000709 \\
\hline & & 0 & 0.001377 & 0.001474 & 0.001633 & 0.001689 & 0.001479 \\
\hline \multirow{8}{*}{10} & \multirow{2}{*}{0.5} & 0.925925 & 0.000490 & 0.000524 & 0.000578 & 0.000590 & 0.000488 \\
\hline & & 0 & 0.001396 & 0.001508 & 0.001711 & 0.001870 & 0.001835 \\
\hline & \multirow{2}{*}{1} & 0.925925 & 0.000490 & 0.000524 & 0.000578 & 0.000590 & 0.000488 \\
\hline & & 0 & 0.001275 & 0.001371 & 0.001543 & 0.001668 & 0.001637 \\
\hline & \multirow[b]{2}{*}{2} & 0.925925 & 0.000490 & 0.000524 & 0.000578 & 0.000590 & 0.000488 \\
\hline & & 0 & 0.001143 & 0.001225 & 0.001368 & 0.001452 & 0.001377 \\
\hline & \multirow[b]{2}{*}{10} & 0.925925 & 0.000490 & 0.000524 & 0.000578 & 0.000590 & 0.000488 \\
\hline & & 0 & 0.001020 & 0.001091 & 0.001208 & 0.001247 & 0.001083 \\
\hline
\end{tabular}

Secondly, the natural frequency $\omega_{m n}$ values (unit $1 / \mathrm{s}$ ) of free vibration ( $\Delta T=0$ ) according to mode shape numbers $m$ and $n$ for the SUS304/Si ${ }_{3} \mathrm{~N}_{4}$ FGM thick circular cylindrical shells are calculated. For the values of fundamental first $(m=n=1)$ natural frequency $\omega_{11}$ vs. $R_{n}$ with $h^{*}=1.2 \mathrm{~mm}$, varied $k_{\alpha}$ and the effects of $c_{1}=0.925925 / \mathrm{mm}^{2}$ and $c_{1}=0 / \mathrm{mm}^{2}$ for $L / h^{*}=5$, 8 and 10 are under $T=1 K, 100 K, 300 K, 600 K$ and $1000 K$ are shown in Table 5 . For the values of natural frequency $\omega_{m n}$ vs. $m, n=1,2, \ldots, 9$ with $R_{n}=0.5, T=300 K, h^{*}=1.2 \mathrm{~mm}$ under varied $k_{\alpha}$ and the effects of $c_{1}=0.925925 / \mathrm{mm}^{2}$ and $c_{1}=0 / \mathrm{mm}^{2}$ for $L / h^{*}=5$ and 10 are shown in Table 6. 
Table 6 Natural frequency $\omega_{m n}$ vs. $m$ and $n$ under varied $k_{\alpha}, c_{1}, R_{n}=0.5$ and $T=300 K$

\begin{tabular}{|c|c|c|c|c|c|c|c|c|c|c|}
\hline \multirow{2}{*}{$\begin{array}{c}c_{1} \\
\left(1 / \mathrm{mm}^{2}\right)\end{array}$} & \multirow{2}{*}{$L / h^{*}$} & \multicolumn{9}{|c|}{$\omega_{1 n}$} \\
\hline & & $n=1$ & $n=2$ & $n=3$ & $n=4$ & $n=5$ & $n=6$ & $n=7$ & $n=8$ & $n=9$ \\
\hline \multirow[t]{2}{*}{0.925925} & 5 & 0.001906 & 0.001347 & 0.001098 & 0.000949 & 0.000847 & 0.000772 & 0.000713 & 0.000666 & 0.000627 \\
\hline & 10 & 00578 & 0.000409 & 0.000334 & 0.000289 & 0.000258 & 0.000236 & 0.000218 & 0.000204 & 0.000193 \\
\hline \multirow{2}{*}{0} & 5 & 05406 & 0.005285 & 0.005244 & 0.005209 & 0.005170 & 0.005126 & 0.005077 & 0.005022 & 0.004963 \\
\hline & 10 & 001711 & 0.001621 & 0.001602 & 0.001595 & 0.001592 & 0.001590 & 0.001588 & 0.001587 & 0.001586 \\
\hline \multirow{2}{*}{$\begin{array}{c}c_{1} \\
\left(1 / \mathrm{mm}^{2}\right)\end{array}$} & \multirow{2}{*}{$L / h^{*}$} & \multicolumn{9}{|c|}{$\omega_{2 n}$} \\
\hline & & $n=1$ & $n=2$ & $n=3$ & $n=4$ & $n=5$ & $n=6$ & $n=7$ & $n=8$ & $n=9$ \\
\hline \multirow{2}{*}{0.925925} & 5 & 0.001347 & 0.000953 & 0.000778 & 0.000673 & 0.000602 & 0.000549 & 0.000508 & 0.000474 & 0.000447 \\
\hline & 10 & 99 & 0.000289 & 0.000 & 0.000204 & 0.000183 & 0.000167 & 0.000154 & 0.000144 & 0.000136 \\
\hline \multirow{2}{*}{0} & 5 & 02712 & 0.002647 & 0.002633 & 0.002626 & 0.002620 & 0.002613 & 0.002606 & 0.002606 & 0.002590 \\
\hline & 10 & 61 & 0.000811 & 0.00 & 0.000797 & 0.000796 & 0.00 & 0.000794 & 0.000794 & 0.00 \\
\hline \multirow{2}{*}{$\begin{array}{c}c_{1} \\
\left(1 / \mathrm{mm}^{2}\right)\end{array}$} & \multirow{2}{*}{$L / h^{*}$} & \multicolumn{9}{|c|}{$\omega_{3 n}$} \\
\hline & & $n=1$ & $n=2$ & $n=3$ & $n=4$ & $n=5$ & $n=6$ & $n=7$ & $n=8$ & $n=9$ \\
\hline \multirow{2}{*}{0.925925} & 5 & .001098 & 0.000778 & 0.00 & 0.000550 & 0.000 & .00 & 0.000415 & 0.000388 & \\
\hline & 10 & 4 & 0.000236 & 0.000192 & 0.000167 & 0.000149 & 0.00 & 0.0 & 0.00 & 0.00 \\
\hline \multirow{2}{*}{0} & 5 & 01817 & 0.001766 & 0.001757 & 0.001753 & 0.001751 & 0.00 & 0.0 & 0.00 & 0.001741 \\
\hline & 10 & 000578 & 0.000 & 0.00 & 0.00 & 0.000531 & 0.00 & 0.0 & 0.00 & 0.00 \\
\hline \multirow{2}{*}{$\begin{array}{c}c_{1} \\
\left(1 / \mathrm{mm}^{2}\right)\end{array}$} & \multirow{2}{*}{$L / h^{*}$} & & & & & $\omega_{4 n}$ & & & & \\
\hline & & $n=1$ & $n=2$ & $n=3$ & $n=4$ & $n=5$ & $n=6$ & $n=7$ & $n=8$ & $n=9$ \\
\hline 0 & 5 & 49 & 0.000673 & 0.000550 & 0.00 & 0.000 & 0.00 & 0.000360 & 0.000337 & 0.000317 \\
\hline 0. & 10 & 89 & 0.000204 & 0.00 & 0.00 & 0.000 & 0.00 & 109 & 0.00 & 0.0 \\
\hline 0 & 5 & & 0.00 & 0.00 & 0.00 & 0.001314 & & 312 & 0.00 & 0.001309 \\
\hline 0 & 10 & 0.00 & 0.000407 & 0.00 & 0.00 & 0.000398 & 0.00 & 0.0 & 0.00 & 0.0 \\
\hline & & & & & & $\omega_{5 n}$ & & & & \\
\hline 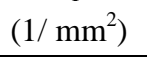 & $L$ & $n=1$ & $n=2$ & $n=3$ & $n=4$ & $n=5$ & $n=6$ & $n=7$ & $n=8$ & $n=9$ \\
\hline 0075025 & 5 & 001 & .000602 & 0.00 & 0.000426 & 0.000 & & 22 & 0.00 & \\
\hline 0.92592 & 10 & 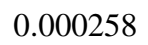 & 00 & 0.00 & 0.00 & 0.000 & 0.0 & 0.0 & 0.00 & \\
\hline & 5 & & & & & 0.00 & & & & \\
\hline 0 & 10 & & & 0.00 & & 0.000318 & & & & \\
\hline & & & & & & $\omega_{6 n}$ & & & & \\
\hline & & $n=1$ & $n=2$ & $n=3$ & $n=4$ & $n=5$ & $n=6$ & $n=7$ & $n=8$ & $n=9$ \\
\hline & 5 & & 49 & & & 0.000 & & & 0.00 & \\
\hline 0.9 & 10 & O & 0.000 & 0.00 & 0.00 & 0.00 & & 89 & & \\
\hline 0 & 5 & & & 0.00 & 0.000 & 0.000877 & & 876 & 0.000875 & 0.000875 \\
\hline & 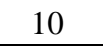 & & 0.00 & 0.00 & 0.00 & 0.000265 & & 265 & 0.00 & 0.000265 \\
\hline 1 & $I$ & & & & & $\omega_{7 n}$ & & & & \\
\hline$\left(1 / \mathrm{mm}^{2}\right)$ & $L$ & & & & $n=$ & $n=5$ & & $n=7$ & $n=$ & $n=9$ \\
\hline 0005025 & & & & & & 0.000 & & & & \\
\hline 0. & 10 & & & & & 0.00 & & & & \\
\hline & 5 & 26 & 0.00 & 0.00 & 0.00 & 0.000751 & & 751 & 0.00 & 0.000750 \\
\hline 0 & 10 & & 0.000234 & 0.000229 & 0.000228 & 0.000227 & 0.000227 & 0.000227 & 0.000227 & 0.000227 \\
\hline$c_{1}$ & & & & & & $\omega_{8 n}$ & & & & \\
\hline$(1 /$ & $n$ & $n=1$ & $n=2$ & $n=3$ & $n=4$ & $n=5$ & $n=$ & $n=7$ & $n=$ & $n=9$ \\
\hline 007 & 5 & & & 0.0 & 0.00 & 0.00 & & 0.0 & 0.00 & \\
\hline $0 . \mathrm{S}^{2}$ & 10 & & & & & & & & & \\
\hline & & 0.000744 & 0.000668 & & 0.000 & 0.000658 & & 557 & 0.000657 & 0.000656 \\
\hline 0 & 10 & & 0.000205 & 0.000201 & 0.000199 & 0.000199 & 0.000199 & 0.000198 & 0.000198 & 0.000198 \\
\hline$c_{1}$ & & & & & & $\omega_{9 n}$ & & & & \\
\hline$(1 / \mathrm{mn}$ & & $n=1$ & $n=2$ & $n=3$ & $n=4$ & $n=5$ & $n=6$ & $n=7$ & $n=8$ & $n=9$ \\
\hline & 5 & 0.000 & 0.000447 & 0.000366 & 0.000 & 0.000 & & 0.000240 & 0.000224 & 0.000212 \\
\hline & 10 & 0.000193 & 0.000156 & 0.000111 & 0.000096 & 0.000087 & 0.000079 & 0.000073 & 0.000069 & 0.000064 \\
\hline 0 & 5 & 0.000684 & 0.000596 & 0.000588 & 0.000586 & 0.000585 & 0.000584 & 0.000584 & 0.000584 & 0.000584 \\
\hline 0 & 10 & 0.000215 & 0.000183 & 0.000179 & 0.000177 & 0.000177 & 0.000176 & 0.000176 & 0.000176 & 0.000176 \\
\hline
\end{tabular}




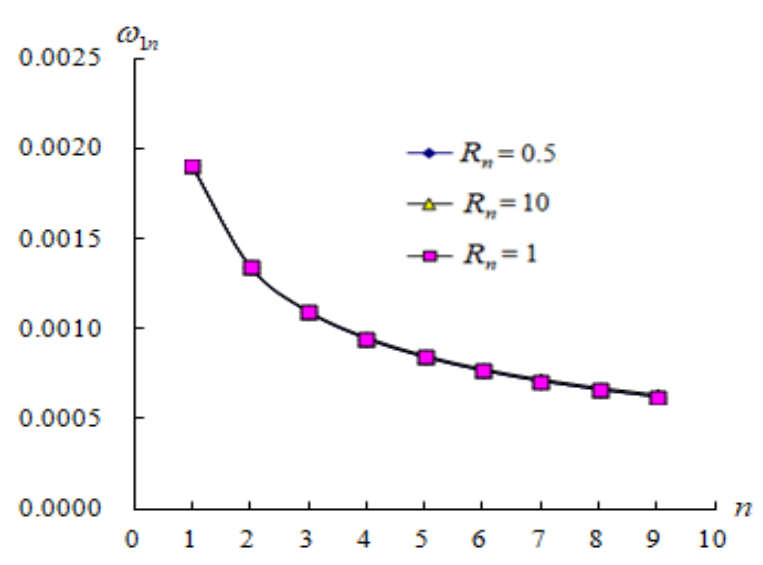

Fig. $2 \omega_{1 n}$ vs. $R_{n}$ for $L / h^{*}=5$

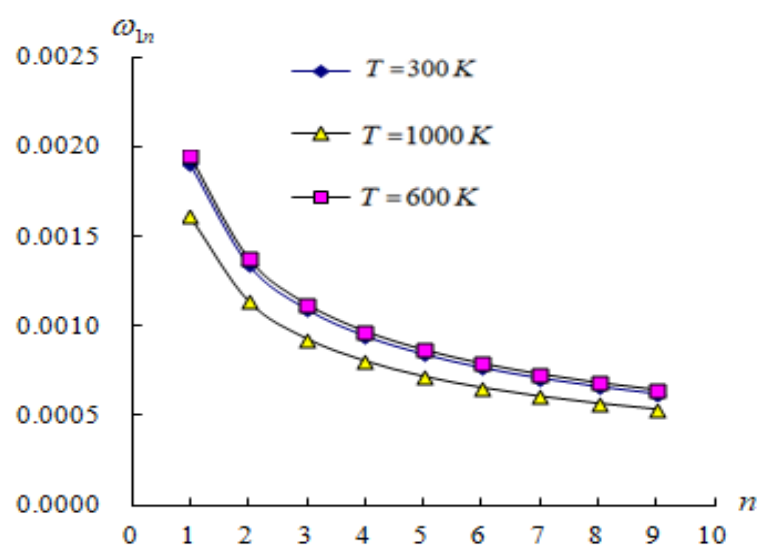

Fig. $4 \omega_{1 n}$ vs. $T$ for $L / h^{*}=5$

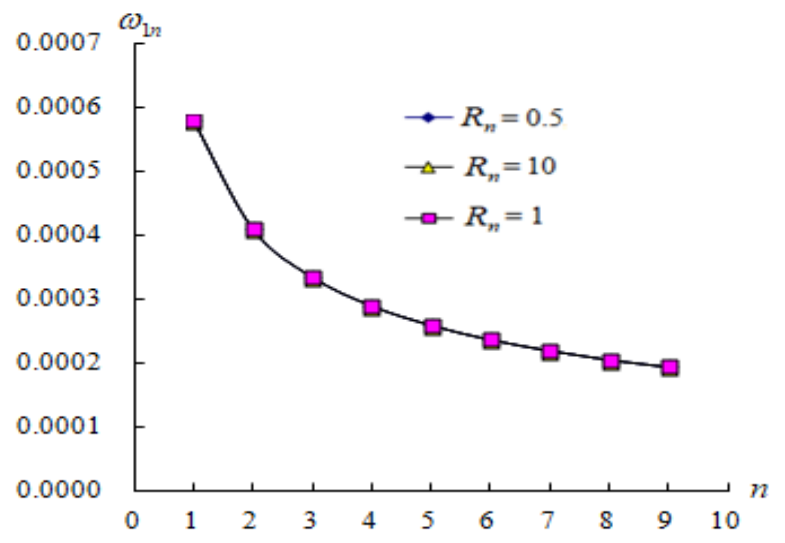

Fig. $3 \omega_{1 n}$ vs. $R_{n}$ for $L / h^{*}=10$

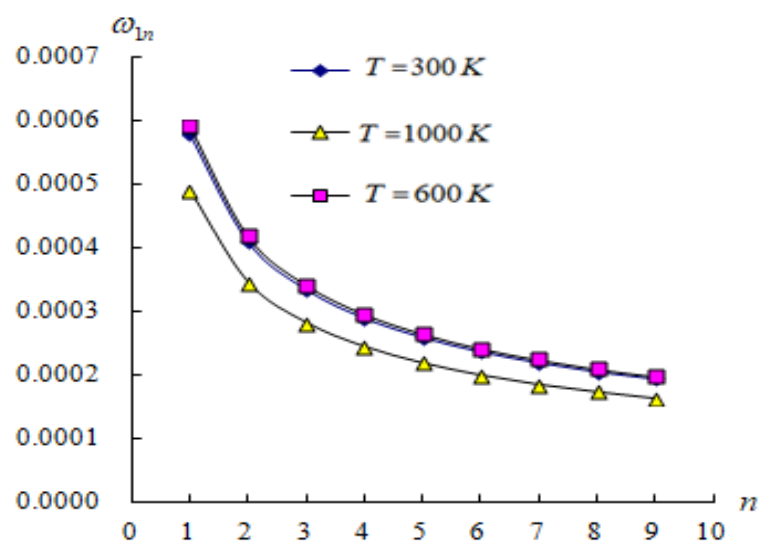

Fig. $5 \omega_{1 n}$ vs. $R_{n}$ for $L / h^{*}=10$

Finally, the natural frequency $\omega_{m n}$ values (unit $\left.1 / \mathrm{s}\right)$ vs. $R_{n}$ and $T$ of free vibration $(\Delta T=0)$ according to mode shape numbers $m=1$ and $n$ (from 1 to 9) for the SUS304/Si ${ }_{3} \mathrm{~N}_{4}$ FGM thick circular cylindrical shells are calculated. Figs. (2)-(3) show the values of $\omega_{1 n}$ vs. $R_{n}$ in FGM circular cylindrical shells for thick $L / h^{*}=5,10$ respectively, with the effects of varied $k_{\alpha}$ and $c_{1}=0.925925 / \mathrm{mm}^{2}$ under $T=300 K$. Generally the values of $\omega_{1 n}$ are decreasing with values of $n$ (from 1 to 9 ) for $L / h^{*}=$ 5, $R_{n}=0.5,1$ and 10. The greatest value of $\omega_{11}=0.00191$ (unit $1 / \mathrm{s}$ ) is found for $L / h^{*}=5$. The values of $\omega_{1 n}$ are also decreasing with values of $n$ (from 1 to 9 ) for $L / h^{*}=10, R_{n}=0.5,1$ and 10. Figs. $4-5$ show the values of $\omega_{1 n}$ vs. $T$ in FGM circular cylindrical shells for thick $L / h^{*}=5,10$ respectively, under the effects of varied $k_{\alpha}, c_{1}=0.925925 / \mathrm{mm}^{2}$ and $R_{n}=0.5$. Generally the values of $\omega_{1 n}$ are decreasing with values of $n$ (from 1 to 9) for $L / h^{*}=5, T=300 K, 600 K$ and $1000 K$, the values of $\omega_{1 n}$ are almost in the same for $T=300 \mathrm{~K}$ and $600 \mathrm{~K}$, but in greater values than that in the $T=1000 \mathrm{~K}$. The greatest value of $\omega_{11}=0.00191$ (unit $1 / \mathrm{s}$ ) is found for $L / h^{*}=5, T=600 \mathrm{~K}$. The values of $\omega_{1 n}$ can stand for higher temperature $T=1000 \mathrm{~K}$ at $L / h^{*}=5$. The values of $\omega_{1 n}$ are decreasing with values of $n$ (from 1 to 9) for $L / h^{*}=10, T=300 K, 600 K$ and $1000 K$, the values of $\omega_{1 n}$ are almost in the same for $T=300 \mathrm{~K}$ and $600 \mathrm{~K}$, but in greater values than that in the $T=1000 \mathrm{~K}$. The greatest value of $\omega_{11}=0.00059$ (unit $1 / \mathrm{s}$ ) is found for $L / h^{*}=10, T=600 \mathrm{~K}$. The values of $\omega_{1 n}$ can stand for higher temperature $T=1000 K$ at $L / h^{*}=10$. The values of $\omega_{1 n}$ at $L / h^{*}=5$ are also found in the greater values than that at $L / h^{*}=10$.

\section{Conclusions}

The values of natural frequency and frequency parameters are calculated and obtained by using the simply homogeneous equation with the polynomial equation in fifth-order of $\lambda_{m n}$ in the free vibration of thick FGM circular cylindrical shells. The 
three items of value effects are considered in nonlinear coefficient term $c_{1}$, shear correction coefficient and environment temperature. Some of the important results are found as follows. (a) Data investigated in the three kinds of frequency parameters under free vibration with and without the effects of $c_{1}$. (b) Generally the values of $\omega_{1 n}$ are decreasing with values of $n$ (from 1 to 9) for $L / h^{*}=5$ and 10, $R_{n}=0.5,1$ and 10. (c) The values of $\omega_{1 n}$ can stand for higher environment temperature $T=1000 K$ at $L / h^{*}=10$. (d) The values of $\omega_{1 n}$ vs. environment temperature $T$ at $L / h^{*}=5$ are found in the greater values than that at $L / h^{*}=10$.

\section{Conflicts of Interest}

The authors declare no conflict of interest.

\section{References}

[1] A. Shahbaztabar, A. Izadi, M. Sadeghian, and M. Kazemi, "Free vibration analysis of FGM circular cylindrical shells resting on the Pasternak foundation and partially in contact with stationary fluid," Applied Acoustics, vol. 153, pp. 87-101, 2019.

[2] A. Zippo, M. Barbieri, and F. Pellicano, "Temperature gradient effect on dynamic properties of a polymeric circular cylindrical shell,” Composite Structures, vol. 216, pp. 301-314, 2019.

[3] A. K. Baltacıŏlu and Ö. Civalek, "Vibration analysis of circular cylindrical panels with CNT reinforced and FGM composites," Composite Structures, vol. 202, pp. 374-388, 2018.

[4] J. Torabi and R. Ansari, "A higher-order isoparametric superelement for free vibration analysis of functionally graded shells of revolution,” Thin-Walled Structures, vol. 133, pp. 169-179, 2018.

[5] A. Nasirmanesh and S. Mohammadi, "An extended finite element framework for vibration analysis of cracked FGM shells,” Composite Structures, vol. 180, pp. 298-315, 2017

[6] Y. Wang and D.Wu, "Free vibration of functionally graded porous cylindrical shell using a sinusoidal shear deformation theory," Aerospace Science and Technology, vol. 66, pp. 83-91, 2017.

[7] F. A. Fazzolari and E. Carrera, "Refined hierarchical kinematics quasi-3D Ritz models for free vibration analysis of doubly curved FGM shells and sandwich shells with FGM core,” Journal of Sound and Vibration, vol. 333, pp. 1485-1508, 2014.

[8] N. Fantuzzi, S. Brischetto, F. Tornabene, and E. Viola, "2D and 3D shell models for the free vibration investigation of functionally graded cylindrical and spherical panels," Composite Structures, vol. 154, pp. 573-590, 2016.

[9] T. Q. Bui, T. V. Do, L. H. T. Ton, D. H. Doan, S. Tanaka, D. T. Pham et al., "On the high temperature mechanical behaviors analysis of heated functionally graded plates using FEM and a new third-order shear deformation plate theory," Composites Part B, vol. 92, pp. 218-241, 2016.

[10] T. V. Do, D. K. Nguyen, N. D. Duc, D. H. Doan, and T. Q. Bui, “Analysis of bi-directional functionally graded plates by FEM and a new third-order shear deformation plate theory,” Thin-Walled Structures, vol. 119, pp. 687-699, 2017.

[11] T. V. Vu, A. Khosravifard, M. R. Hematiyan, and T. Q. Bui, "A new refined simple TSDT-based effective meshfree method for analysis of through-thickness FG plates,” Applied Mathematical Modelling, vol. 57, pp. 514-534, 2018.

[12] W. Zhang, Y. X. Hao, and J. Yang, "Nonlinear dynamics of FGM circular cylindrical shell with clamped-clamped edges," Composite Structures, vol. 94, pp. 1075-1086, 2012.

[13] H. L. Dai, Y. N. Rao, and Ting Dai, "A review of recent researches on FGM cylindrical structures under coupled physical interactions, 2000-2015," Composite Structures, vol. 152, pp. 199-225, 2016.

[14] R. Ansari and M. Darvizeh, "Prediction of dynamic behaviour of FGM shells under arbitrary boundary conditions," Composite Structures, vol. 85, pp. 284-292, 2008.

[15] C. C. Hong, "Effects of varied shear correction on the thermal vibration of functionally-graded material shells in an unsteady supersonic flow," Aerospace, vol. 4, pp. 1-15, 2017.

[16] C. C. Hong, "The GDQ method of thermal vibration laminated shell with actuating magnetostrictive layers," International Journal of Engineering and Technology Innovation, vol. 7, pp. 188-200, 2017.

[17] C. C. Hong, "Thermal vibration of magnetostrictive functionally graded material shells," European Journal of Mechanics A/Solids, vol. 40, pp. 114-122, 2013. 
[18] C. C. Hong, "Rapid heating induced vibration of magnetostrictive functionally graded material plates," Transactions of the ASME, Journal of Vibration and Acoustics, vol. 134, 021019, pp. 1-11, 2012.

[19] S. J. Lee, J. N. Reddy, and F. Rostam-Abadi, "Transient analysis of laminated composite plates with embedded smart-material layers," Finite Elements in Analysis and Design, vol. 40, pp. 463-483, 2004.

[20] S. J. Lee and J. N. Reddy, "Non-linear response of laminated composite plates under thermomechanical loading," International Journal of Non-Linear Mechanics, vol. 40, pp. 971-985, 2005.

[21] J. M. Whitney, "Structural analysis of laminated anisotropic plates," Lancaster: Pennsylvania, USA, Technomic Publishing Company, Inc., 1987.

[22] J. N. Reddy, "Energy principles and variational methods in applied mechanics," Wiley, New York, 2002.

[23] C. C. Hong, "Thermal vibration of magnetostrictive functionally graded material shells by considering the varied effects of shear correction coefficient," International Journal of Mechanical Sciences, vol. 85, pp. 20-29, 2014.

[24] H. A. Sepiani, A. Rastgoo, F. Ebrahimi, and A. G. Arani, "Vibration and buckling analysis of two-layered functionally graded cylindrical shell considering the effects of transverse shear and rotary inertia," Materials and Design, vol. 31, pp. 1063-1069, 2010.

[25] H. Chen, A. Wang, Y. Hao, and W. Zhang, "Free vibration of FGM sandwich doubly-curved shallow shell based on a new shear deformation theory with stretching effects,” Composite Structures, vol.179, pp. 50-60, 2017.

Copyright $\odot$ by the authors. Licensee TAETI, Taiwan. This article is an open access article distributed under the terms and conditions of the Creative Commons Attribution (CC BY-NC) license (https://creativecommons.org/licenses/by-nc/4.0/). 